\title{
PAX6 missense variants in two families with isolated foveal hypoplasia and nystagmus: evidence of paternal postzygotic mosaicism
}

\author{
Dulce Lima Cunha $\mathbb{1}^{1} \cdot$ Nicholas Owen $\mathbb{E}^{1,2} \cdot$ Vijay Tailor $\mathbb{D}^{2,3} \cdot$ Marta Corton $^{4,5} \cdot$ Maria Theodorou $^{2}$. \\ Mariya Moosajee $\mathbb{I I}^{1,2,6}$
}

Received: 16 April 2020 / Revised: 15 September 2020 / Accepted: 22 September 2020 / Published online: 6 October 2020

(c) The Author(s) 2020. This article is published with open access

\begin{abstract}
PAX6 is considered the master regulator of eye development, the majority of variants affecting this gene cause the panocular developmental eye disorder aniridia. Although no genotype-phenotype correlations are clearly established, missense variants affecting the DNA-binding paired domain of PAX6 are usually associated with non-aniridia phenotypes like microphthalmia, coloboma or isolated foveal hypoplasia. In this study, we report two missense heterozygous variants in the paired domain of PAX6 resulting in isolated foveal hypoplasia with nystagmus in two independent families: c.112 C $>\mathrm{G}$; p.(Arg38Gly) and c.214 G >C; p.(Gly72Arg) in exons 5 and 6, respectively. Furthermore, we provide evidence that paternal postzygotic mosaicism is the cause of inheritance, with clinically unaffected fathers and reduced affected allele fraction. This work contributes to increase the phenotypic spectrum caused by PAX6 variants, and to our knowledge, is the first report to describe the presence of postzygotic parental mosaicism causing isolated foveal hypoplasia with nystagmus. These results support the growing evidence that suggest an overestimation of sporadic cases with PAX6 variants, which has strong implications for both genetic counselling and family planning.
\end{abstract}

These authors contributed equally: Dulce Lima Cunha, Nicholas Owen

Supplementary information The online version of this article (https:// doi.org/10.1038/s41431-020-00737-1) contains supplementary material, which is available to authorized users.

Mariya Moosajee

m.moosajee@ucl.ac.uk

1 UCL Institute of Ophthalmology, London, UK

2 Moorfields Eye Hospital NHS Foundation Trust, London, UK

3 UCL Experimental Psychology, London, UK

4 Department of Genetics \& Genomics, Instituto de Investigación Sanitaria-Fundación Jiménez Díaz University Hospital Universidad Autónoma de Madrid (IIS-FJD, UAM), Madrid, Spain

5 Centre for Biomedical Network Research on Rare Diseases (CIBERER), Madrid, Spain

6 Great Ormond Street Hospital for Children NHS Foundation Trust, London, UK

\section{Introduction}

PAX6 is a member of the highly conserved paired-box (PAX) family of transcription factors and is critical for neural and ocular development [1]. The PAX6 gene (OMIM 607108) is composed of 14 exons that encode a protein containing two DNA-binding domains, the paired domain (PD) and the homeodomain, followed by a proline-serinethreonine rich domain with transactivation properties [2]. Variants in PAX6 have been reported across the coding sequence of the gene, the majority being loss of function [3, 4]. PAX6 haploinsufficiency tends to cause aniridia (OMIM 106210), a pan-ocular disorder characterised by partial or complete iris hypoplasia, foveal hypoplasia and nystagmus, with subsequent development of cataracts, glaucoma and corneal keratopathy [5, 6]. In contrast, missense variants in PAX6 are mostly concentrated in exons 5 and 6, which encode for the PD, and are largely associated with non-aniridia phenotypes such as microphthalmia, ocular coloboma (OMIM 120200), foveal hypoplasia with or without anterior segment anomalies and/or cataract (OMIM 136520) [3, 7]. PAX6 variants are associated with high phenotypic variability, complicating the establishment 
of genotype-phenotype correlations and hindering clinical diagnosis and management [6].

In autosomal dominant disorders, it is estimated that parental mosaicism can be found in up to $17 \%$ of sporadic cases with apparent de novo variants [8]. In patients with PAX6-related aniridia, two-thirds are familial cases, while the remaining are sporadic comprising of de novo point variants or, less frequently, larger deletions encompassing $P A X 6$ and the neighbouring gene WT1, resulting in Wilms tumour, aniridia, genitourinary anomalies and mental retardation (WAGR) syndrome [5, 9]. The presence of parental mosaicism in three $P A X 6$-affected individuals (two with aniridia and one with microphthalmia) was recently reported by Tarilonte et al., where affected allele fractions in unaffected or mildly affected fathers ranged from 10 to $30 \%$ in different tissues analysed [10]. In this study, we report two independent families presenting with isolated foveal hypoplasia and nystagmus caused by missense heterozygous changes in PAX6 predicted to affect the PD. Furthermore, we show that the presence of paternal postzygotic mosaicism is the cause of inheritance.

\section{Material and methods}

\section{Patient description}

This study was approved by Moorfields Eye Hospital and the National Research Ethics Committee and was conducted in adherence to the tenets of the Declaration of Helsinki; informed written consent was obtained from all participants. Ophthalmic evaluation included full orthoptic assessment, refraction, best corrected visual acuity using LogMAR, slit lamp examination, and fundus examination recorded with anterior segment and fundus colour imaging. Investigations included eye movement recordings (EMR), electrophysiology, spectral domain optical coherence tomography (SD-OCT) and fundus autofluorescence.

\section{Molecular screening}

Sanger sequencing of the probands DNA was performed to screen the PAX6 gene at the NHS Wessex Regional Genetics Laboratory (WRGL). PAX6 transcript NM_000280.4/ ENST00000643871.1 was used for variant nomenclature and exon numbering. The gnomAD data set (https://gnomad.broadinstitute.org/; accessed Nov 2019) was used to estimate the prevalence of the identified variants. Deleteriousness was scored using in silico algorithms SIFT, PolyPhen2, MutationTaster, PROVEAN and CADD scoring [11]. Variants were submitted to ClinVar with accession numbers VCV000800413.1 and VCV000637045.2. Siblings and parents had familial testing to confirm segregation. Urine, buccal swab and hair follicles were further collected from the fathers of both probands and genomic DNA extracted with QiAmp DNA MicroKit (Qiagen, Germany), amplified by PCR using standard conditions and variants were confirmed by direct sequencing. Droplet digital PCR (ddPCR) analysis was performed in DNA from blood obtained from Family 1 father (1-2) and unaffected sibling (1-4) using a customdesigned TaqMan SNP Genotyping Assay (Applied Biosystems, CA, USA) for the variant NM_000280.4:c.214 G > C as previously described [10].

\section{Results}

\section{Clinical findings}

Two unrelated families were identified (Fig. 1a, b), where the probands (1-3 and 2-4) exhibited nystagmus and reduced vision from infancy. Family 1 were of British Caucasian descent, and Family 2 were Somalian, neither were consanguineous. Detailed ophthalmological features of probands and family members are presented in Table 1 and Fig. 1. The best corrected visual acuity ranged between 0.00 and 1.00 LogMAR in molecularly confirmed affected members. There was significant intra-familial phenotypic variability with patient 2-3 from Family 2 being more mildly affected than his siblings. All affected patients had a refractive error varying between mild-moderate hypermetropic to myopic astigmatism. SD-OCT of affected patients revealed variable degrees of foveal hypoplasia, graded according to Thomas et al. [8]. None had any iris abnormalities, anterior segment dysgenesis or signs of cataract. No systemic abnormalities were present.

\section{Genetic analysis}

Genotyping of proband 1-3 from Family 1 revealed a heterozygous missense variant in exon 6 of PAX6, NM_000280.4:c.214 G > C; p.(Gly72Arg) (Fig. 2a). DNA analysis of proband 2-4 from Family 2 showed a heterozygous missense variant in exon 5 of PAX6, NM_000280.4: c.112 C > G; p.(Arg38Gly) (Fig. 2c). Both variants are predicted pathogenic (Supplementary Table 1); the probands from each family were included in a recent report of genotypes solved by the WRGL [12]. Neither of the probands' parents presented with any ocular abnormalities or visual dysfunction; the mothers were homozygous for the normal allele. However, a second low peak with the respective affected allele was visible in the sequences of both fathers: $\mathrm{G}>\mathrm{C}$ in 1-2 (Family 1) and $\mathrm{C}>\mathrm{G}$ in 2-1 (Family 2), pointing to postzygotic mosaicism in these individuals (Fig. 2a, c). 
A

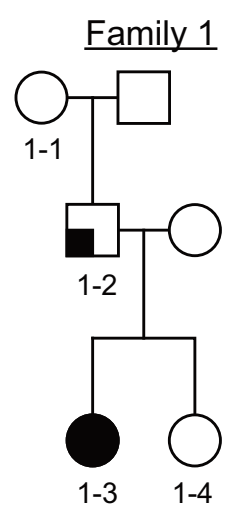

B

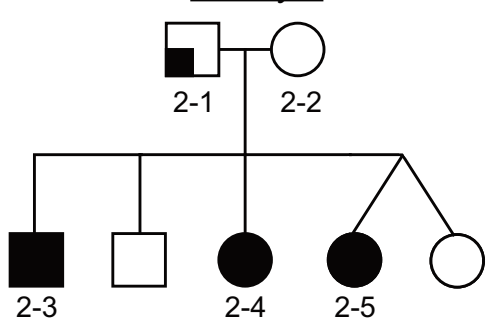

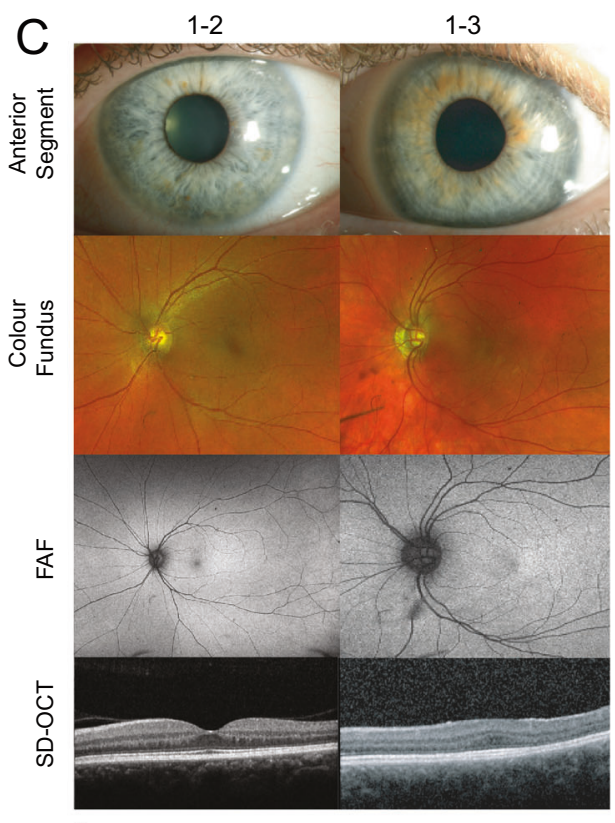

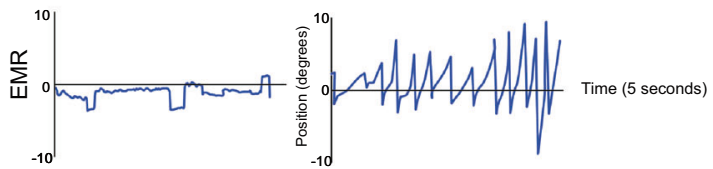

$2-4$
$2-5$

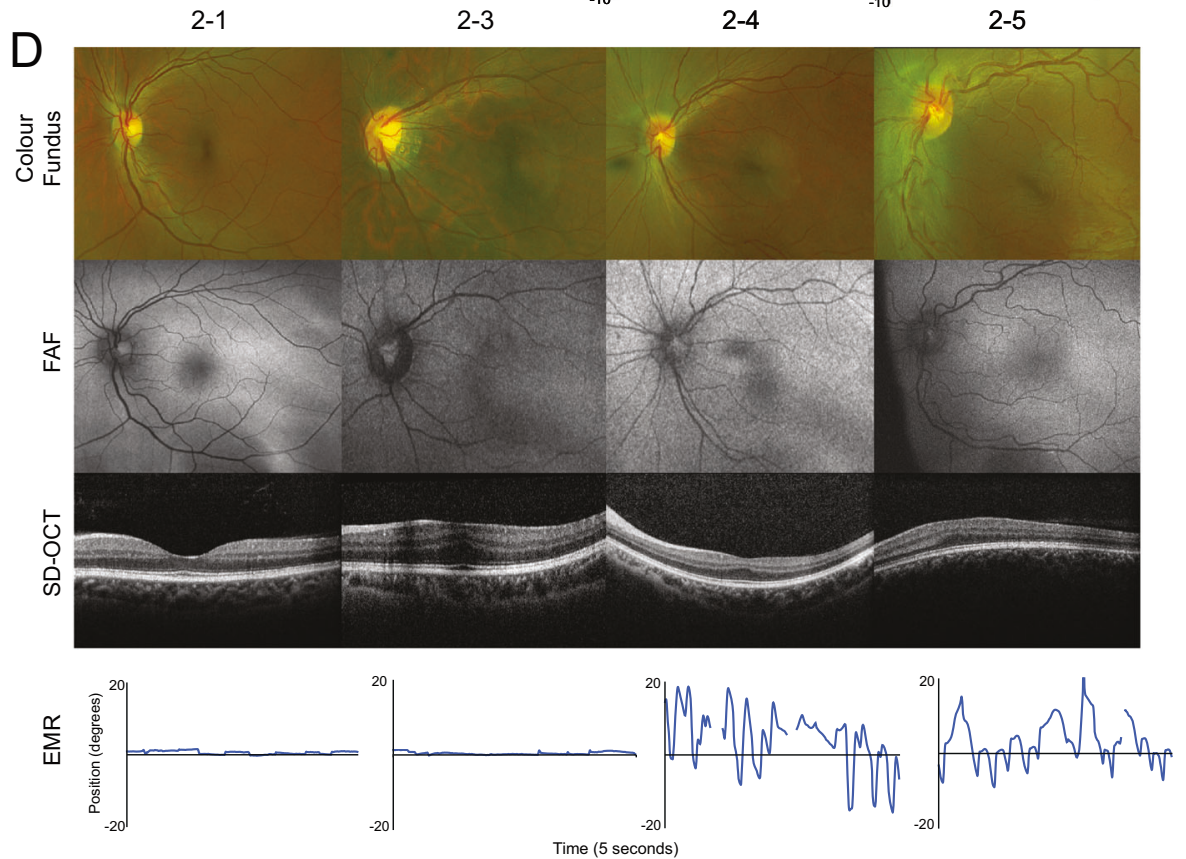

Fig. 1 Pedigree and clinical imaging of probands and members of Families 1 and 2 displaying the left eye only (although there was symmetry between both eyes). a Three-generation Caucasian British non-consanguineous pedigree, with one affected patient (proband 1-3). b Two-generation Somalian non-consanguineous pedigree with three affected siblings (proband 2-4). c Family 1, father (1-2) and

\section{Evidence of parental mosaicism}

To further investigate the presence of mosaicism in individuals 1-2 and 2-1 we analysed DNA extracted from urine proband (1-3) showing anterior segment photos with normal iris architecture, colour fundus image, fundus autofluorescence (FAF), spectral domain optical coherence tomography (SD-OCT) and eye movement recordings (EMR). d Family 2 with father (2-1), eldest brother (2-3), proband (2-4) and younger sister (2-5) showing colour fundus, FAF, SD-OCT and EMR.

epithelial cells, hair follicles and buccal epithelial cells. Sanger sequencing confirmed the presence of a smaller peak corresponding to the affected allele in all samples tested (Fig. 2b, d). ddPCR was then performed to quantify the 


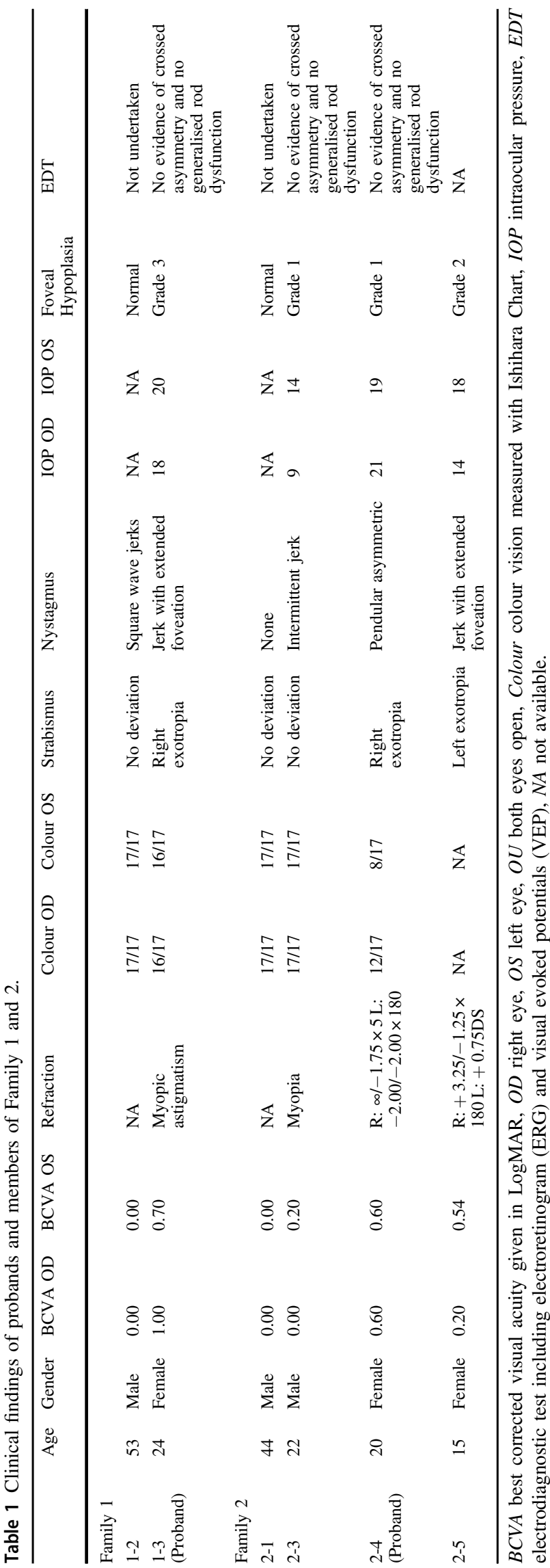

fraction of the affected allele using DNA from the blood of individual 1-2 (Family 1). ddPCR could not be performed for individual 2-1 (Family 2), due to repeated QC failure during synthesis of the Taqman assay for the variant NM_000280.4:c.112 C>G. Analysis of individual 1-2 confirmed the presence of mosaicism, showing an affected allele fraction of $30.4 \%$ (Fig. 2e). DNA from the proband's unaffected sister (1-4) was used as a control and shows no detection of affected allele (Fig. 2f).

\section{Discussion}

We report two families with isolated foveal hypoplasia and nystagmus (OMIM 136520) caused by two different PAX6 missense variants predicted to affect the PD [12]. Reported variants affecting the amino acid Gly72 are largely associated with nystagmus and foveal hypoplasia (c.214 G > A, p.(Gly72Ser) and c.214 G > T, p.(Gly72Cys)) [12, 13], and combined with high myopia (c.215 G $>$ T, p.(Gly72Val)) [14]. For the amino acid Arg38, variants have been associated with nystagmus and congenital cataracts, or bilateral microphthalmia (c.113 G > A, p.(Arg38Gln)) [15, 16]; c.112 C > T, p.(Arg38Trp) has been identified in patients with nystagmus, foveal hypoplasia, microcornea and optic nerve hypoplasia [17, 18] or iris coloboma with cataracts [16]. Our findings support the evidence that PD missense variants, particularly in the C-terminal subdomain of the $\mathrm{PD}$, are more frequently associated with non-aniridia phenotypes [3, 4]. These variable phenotypes are likely due to the physicochemical differences of the resulting amino acid and its effect in the DNA-binding activity of the PD [19]. However, it has recently been noted that some missense changes are likely to affect splicing mechanisms, which could in fact result in loss of function variants, perhaps explaining the cases presenting with iris phenotype [20].

Germline or postzygotic mosaicism has long been suspected to be a cause for apparent de novo cases with PAX6 variants [21, 22], but only recently it was confirmed by analysis of different somatic tissues of suspected carriers [10]. We report the presence of postzygotic mosaicism in two clinically unaffected individuals, with father 1-2 carrying an estimated affected allele fraction of $30.4 \%$, which combined with the presence in different tissues, points to an early postzygotic event. EMR detected square wave jerks at a frequency higher in the otherwise unaffected father 1-2 compared to normal unaffected individuals, which could suggest the affected allele is also present in his cells of ocular origin, resulting in a mild defect. Since PAX6 is a dose-sensitive gene, it could be hypothesised that if enough protein could be restored to a level equivalent to $70 \%$ wildtype allele, this could be enough to alleviate clinical disease. This may be possible through strategies such as nonsense suppression or gene augmentation. 
A

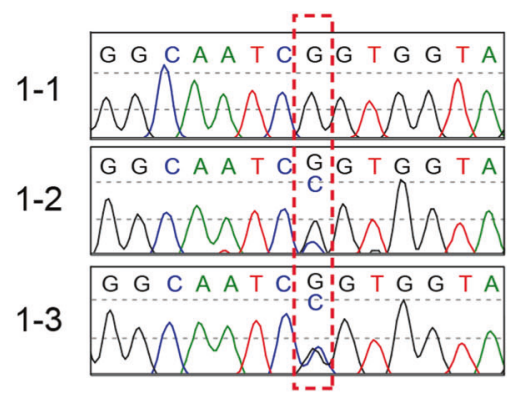

PAX6, c.214G>C, p.(Gly72Arg)
B

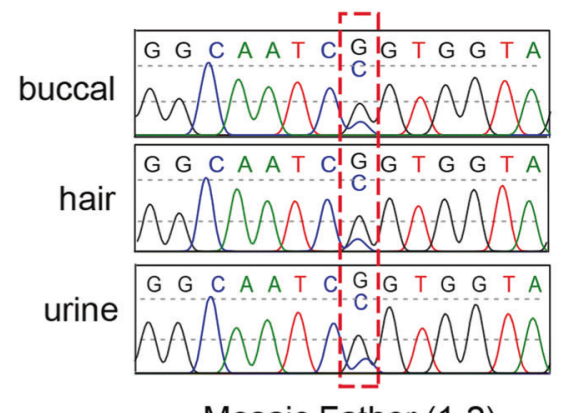

Mosaic Father (1-2)

PAX6, c.214G>C, p.(Gly72Arg)

C
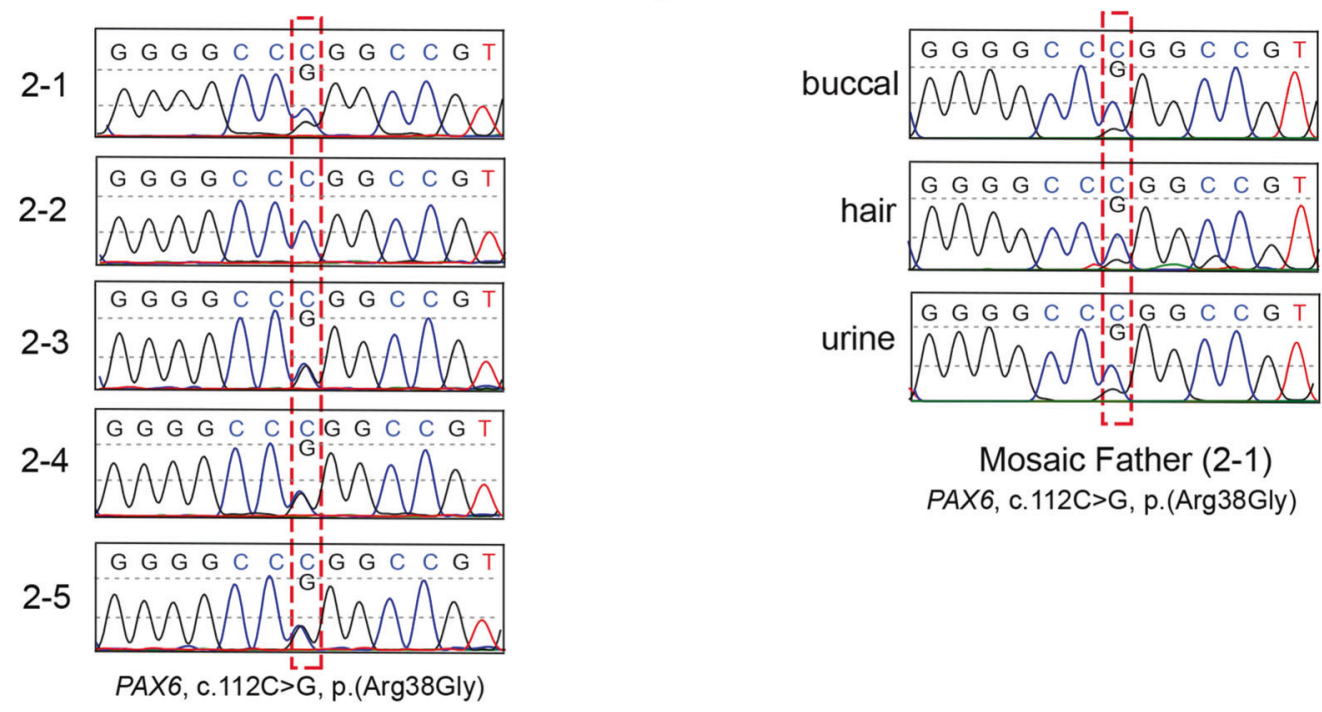

Mosaic Father (2-1)

$P A X 6$, c. 112C $>$ G, p. (Arg38Gly)
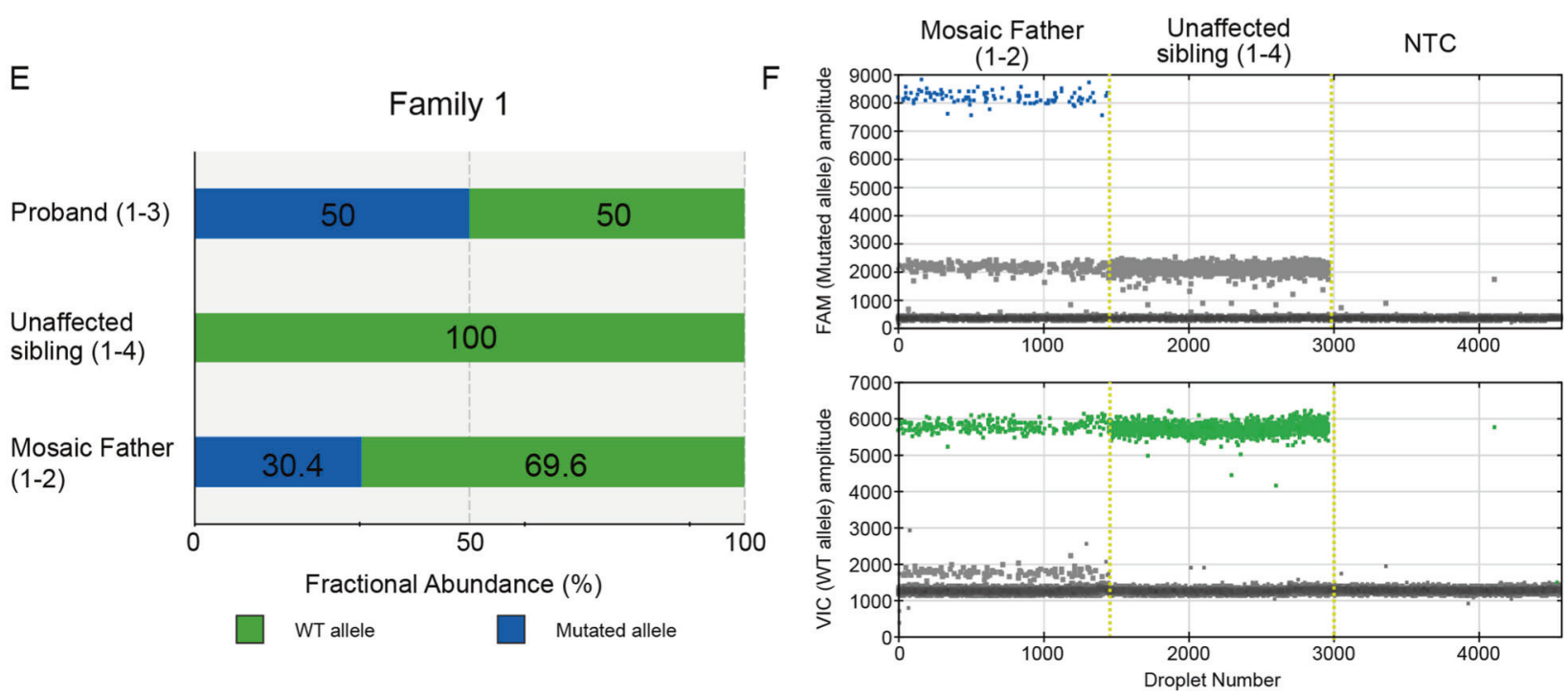

To our knowledge, this is the first report of paternal mosaicism involving PAX6 variants causing isolated foveal hypoplasia with nystagmus. This work further supports the

underestimation of mosaicism rates in $P A X 6$ sporadic cases, which has important implications for genetic counselling, family planning and disease management. Until the genetic 
Fig. 2 Genotype information of probands and members of Families 1 and 2. a DNA chromatograms from blood of all the tested members of Family 1, the position of the variant (c. $214 \mathrm{G}>\mathrm{C}$ ) in exon 6 of PAX6 bounded by the red dotted box. b DNA chromatograms from the father (1-2) of the proband from Family 1 from somatic tissues: buccal, hair and urine epithelia. c DNA chromatograms from blood samples from tested members of Family 2 highlighting PAX6 c. $112 \mathrm{C}>\mathrm{G}$ variant within the red dotted box. d DNA chromatograms from the father $(2-1)$ of the proband from Family 2 from somatic tissues: buccal, hair and urine epithelia. e Quantification of allele abundance by Digital Droplet PCR (ddPCR) for Family 1. Allele percentages of suspected mosaic father (1-2), non-carrier sibling (1-4) and proband (1-3). f Fluorescence amplitude plot showing presence of FAM-labelled droplets in blue (affected allele) in mosaic father (1-2) and absent in non-carrier sibling (1-4, wildtype) as well as the negative control (no template control, NTC). VIC-labelled droplets (green), containing the wildtype allele, were detected in both individuals. Unlabelled droplets are represented in grey.

diagnosis is confirmed in patients, sporadic cases must be investigated for WAGR syndrome with serial renal ultrasound monitoring of potential Wilms tumour. The presence of paternal mosaicism highlights the importance of performing genetic screening of healthy parents of sporadic cases, since there could be a higher recurrence risk in future offspring.

Acknowledgements We thank the families for their involvement in this study.

Funding MM is supported by the Wellcome Trust (205174/Z/16/Z), National Institute for Health Research (NIHR) Biomedical Research Centre at Moorfields Eye Hospital NHS Foundation Trust and UCL Institute of Ophthalmology, Fight for Sight UK, Aniridia Network and Moorfields Eye Charity. MC is supported by the Spanish Institute of Health Carlos III (ISCIII)/European Regional Development Fund (ERDF) (PI17_01164 and CPII17_00006), the Regional Government of Madrid (CAM, B2017/BMD3721) and Spanish Federation of Rare Diseases (FEDER).

\section{Compliance with ethical standards}

Conflict of interest The authors declare that they have no conflict of interest.

Publisher's note Springer Nature remains neutral with regard to jurisdictional claims in published maps and institutional affiliations.

Open Access This article is licensed under a Creative Commons Attribution 4.0 International License, which permits use, sharing, adaptation, distribution and reproduction in any medium or format, as long as you give appropriate credit to the original author(s) and the source, provide a link to the Creative Commons license, and indicate if changes were made. The images or other third party material in this article are included in the article's Creative Commons license, unless indicated otherwise in a credit line to the material. If material is not included in the article's Creative Commons license and your intended use is not permitted by statutory regulation or exceeds the permitted use, you will need to obtain permission directly from the copyright holder. To view a copy of this license, visit http://creativecommons. org/licenses/by/4.0/.

\section{References}

1. Jordan T, Hanson I, Zaletayev D, Hodgson S, Prosser J, Seawright A, et al. The human PAX6 gene is mutated in two patients with aniridia. Nat Genet. 1992;1:328-32.

2. Glaser T, Walton DS, Maas RL. Genomic structure, evolutionary conservation and aniridia mutations in the human PAX6 gene. Nat Genet. 1992;2:232-9.

3. Lima Cunha D, Arno G, Corton M, Moosajee M. The spectrum of PAX6 mutations and genotype-phenotype correlations in the eye. Genes. 2019;10:1050.

4. Tzoulaki I, White IM, Hanson IM. PAX6 mutations: genotypephenotype correlations. BMC Genet. 2005;6:27.

5. Hingorani M, Hanson I, van Heyningen V. Aniridia. Eur J Hum Genet. 2012;20:1011-7.

6. Moosajee M, Hingorani M, Moore AT PAX6-Related Aniridia. In: Adam MP, Ardinger HH, Pagon RA, Wallace SE, Bean LJH, Stephens K, et al., editors. GeneReviews $\left({ }^{\circledR}\right)$. Seattle (WA): University of Washington, Seattle Copyright (C) 1993-2020, University of Washington, Seattle. GeneReviews is a registered trademark of the University of Washington, Seattle. All rights reserved.; 2018.

7. Thomas S, Thomas MG, Andrews C, Chan WM, Proudlock FA, McLean RJ, et al. Autosomal-dominant nystagmus, foveal hypoplasia and presenile cataract associated with a novel PAX6 mutation. Eur J Hum Genet. 2014;22:344-9.

8. Acuna-Hidalgo R, Bo T, Kwint MP, van de Vorst M, Pinelli M, Veltman JA, et al. Post-zygotic Point Mutations Are an Underrecognized Source of De Novo Genomic Variation. Am J Hum Genet. 2015;97:67-74.

9. Moosajee M, Hingorani M, Moore AT PAX6-Related Aniridia. In: Adam MP, Ardinger HH, Pagon RA, Wallace SE, Bean LJH, Stephens K, et al., editors. GeneReviews((R)). Seattle (WA)2018.

10. Tarilonte M, Morín M, Ramos P, Galdós M, Blanco-Kelly F, Villaverde $\mathrm{C}$, et al. Parental mosaicism in PAX6 causes intrafamilial variability: implications for genetic counseling of congenital aniridia and microphthalmia. Front Genet. 2018;9:479.

11. Liu X, Jian X, Boerwinkle E. dbNSFP: a lightweight database of human nonsynonymous SNPs and their functional predictions. Hum Mutat. 2011;32:894-9.

12. Cross E, Duncan-Flavell PJ, Howarth RJ, Crooks RO, Thomas NS, Bunyan DJ. Screening of a large PAX6 cohort identified many novel variants and emphasises the importance of the paired and homeobox domains. Eur J Med Genet. 2020; 63:103940.

13. Hingorani M, Williamson KA, Moore AT, van Heyningen V. Detailed ophthalmologic evaluation of 43 individuals with PAX6 mutations. Investigative Ophthalmol Vis Sci. 2009;50:2581-90.

14. Smirnov VM, Calvas P, Drumare I, Marks C, Defoort-Dhellemmes $\mathrm{S}$. Extreme myopia in a family with a missense PAX6 mutation: extended phenotype. Ophthalmic Genet. 2019;40:64-5.

15. Li J, Leng Y, Han S, Yan L, Lu C, Luo Y, et al. Clinical and genetic characteristics of Chinese patients with familial or sporadic pediatric cataract. Orphanet J Rare Dis. 2018;13:94.

16. Williamson KA, Hall HN, Owen LJ, Livesey BJ, Hanson IM, Adams GGW, et al. Recurrent heterozygous PAX6 missense variants cause severe bilateral microphthalmia via predictable effects on DNA-protein interaction. Genet Med. 2020;22: 598-609.

17. Solomon BD, Pineda-Alvarez DE, Balog JZ, Hadley D, Gropman AL, Nandagopal R, et al. Compound heterozygosity for mutations in PAX6 in a patient with complex brain anomaly, neonatal diabetes mellitus, and microophthalmia. Am J Med Genet Part A. 2009;149a:2543-6. 
18. Henderson RA, Williamson K, Cumming S, Clarke MP, Lynch SA, Hanson IM, et al. Inherited PAX6, NF1 and OTX2 mutations in a child with microphthalmia and aniridia. Eur J Hum Genet. 2007;15:898-901.

19. Lee S, Lee SH, Heo H, Oh EH, Shin JH, Kim HS, et al. Impaired DNA-binding affinity of novel PAX6 mutations. Sci Rep. 2020; 10:3062.

20. Vasilyeva TA, Marakhonov AV, Voskresenskaya AA, Kadyshev VV, Käsmann-Kellner B, Sukhanova NV, et al. Analysis of genotype-phenotype correlations in PAX6-associated aniridia.
J Med Genet. 2020. https://doi.org/10.1136/jmedgenet-2019-106172. [Epub ahead of print].

21. Deml B, Reis LM, Lemyre E, Clark RD, Kariminejad A, Semina EV. Novel mutations in PAX6, OTX2 and NDP in anophthalmia, microphthalmia and coloboma. Eur J Hum Genet. 2016;24: 535-41.

22. Riera M, Wert A, Nieto I, Pomares E. Panel-based whole exome sequencing identifies novel mutations in microphthalmia and anophthalmia patients showing complex Mendelian inheritance patterns. Mol Genet Genom Med. 2017;5:709-19. 